\title{
Controlling the energy flow in nonlinear lattices: a model for a thermal rectifier.
}

\author{
M. Terraneo ${ }^{1,3}$, M. Peyrard ${ }^{2}$ and G. Casati ${ }^{1,3,4}$ \\ 1 International Center for the Study of Dynamical Systems, \\ Universitá degli Studi dell'Insubria, Via Valleggio 11, 22100 Como, Italy \\ 2 Laboratoire de Physique, Ecole Normale Supérieure de Lyon, 69364 Lyon cedex 07, France \\ ${ }^{3}$ Istituto Nazionale per la Fisica della Materia, Unitá di Como, Via Valleggio 11, 22100 Como, Italy \\ ${ }^{4}$ Istituto Nazionale di Fisica Nucleare, Sezione di Milano, Via Celoria 16, 20133 Milano, Italy
}

(October 25, 2018)

\begin{abstract}
We address the problem of heat conduction in 1-D nonlinear chains; we show that, acting on the parameter which controls the strength of the on site potential inside a segment of the chain, we induce a transition from conducting to insulating behavior in the whole system. Quite remarkably, the same transition can be observed by increasing the temperatures of the thermal baths at both ends of the chain by the same amount. The control of heat conduction by nonlinearity opens the possibility to propose new devices such as a thermal rectifier.
\end{abstract}

In recent years a renewed attention has been directed to the energy transport in dynamical systems, a problem which has been denoted by Peierls as one of the outstanding unsolved problems of modern physics [1]. These efforts mainly focused on the possibility to derive the Fourier law of heat conduction on purely dynamical grounds without recourse to any statistical assumption [2 11]. In spite of relevant progresses, several problems remain open and we are still far from a complete understanding [5 11.

In this paper we investigate a different and important problem namely the possibility to control the energy transport inside a nonlinear 1D chain connecting two thermostats at different temperatures. We show that we can parametrically control the heat flux through the system by acting on a small central part of the chain. Even more interestingly, we show that it is possible to adjust the heat flux by varying the temperatures of both thermostats, keeping constant the temperature difference. Thus we provide a simple mechanism to change the properties of the system, from a normal conductor obeying Fourier law, down to an almost perfect insulator. Controlling heat conduction by nonlinearity opens new possibilities, such as the design of a lattice that carries heats preferentially in one direction, i.e. a thermal rectifier.

We consider the Hamiltonian

$$
H=\sum_{n=1, N} \frac{p_{n}^{2}}{2 m}+V_{n}\left(y_{n}\right)+\frac{1}{2} K\left(y_{n}-y_{n-1}\right)^{2}
$$

which describes a chain of $N$ particles with harmonic coupling of constant $K$ and a Morse on-site potential $V_{n}\left(y_{n}\right)=D_{n}\left(e^{-\alpha_{n} y_{n}}-1\right)^{2}$. This model was introduced for DNA chains where $m$ is the reduced mass of a base pair, $y_{n}$ denotes the stretching from equilibrium position of the hydrogen bonds connecting the two bases of the $n$-th pair and $p_{n}$ is its momentum 12] 14. In the context of the present study, model (11) can simply be viewed as a generic system of anharmonic coupled oscillators, the onsite potential arising from interactions with other parts of the system, not included in the model. The Morse potential is simply an example of a highly anharmonic soft potential, which has a frequency that decreases drastically when the amplitude of the motion increases.

In this paper we consider the out-of-equilibrium properties of model (11) by numerically simulating the dynamics of the $N$ particle chain, coupled, at the two ends, with thermal baths at different temperatures $T_{1}$ and $T_{2}$. We thermalize at $T_{1}$ and $T_{2}$ the first and the last $L$ particles by using Nosé-Hoover thermostats chains [15]16], or a Langevin description when we investigate cases very far from equilibrium. We then integrate the differential equations of motion both for the thermostats and for the chain with a 4th order Runge-Kutta method as described in 17]. The baths temperatures $T_{1}, T_{2}$ are never large enough to drive the system beyond the thermal denaturation temperature $T_{c}$ above which the mean value of $y_{n}$ diverges [14].

We compute the temperature profile inside the system, i.e. the local temperature at site $n$ defined as $T_{n}=m\left\langle\dot{{y_{n}}^{2}}\right\rangle$, where \langle\rangle stands for temporal average, and the local heat flux $J_{n}=K\left\langle\dot{y}_{n}\left(y_{n}-y_{n-1}\right)\right\rangle$ [18]. The simulations are performed long enough to allow the system to reach a non-equilibrium steady state with local thermal equilibrium where the local heat flux is constant along the chain.

As a preliminary step we have considered the homogeneous case in which $D_{n}=D, \alpha_{n}=\alpha, n=1, . . N$. Here, as expected, we have detected a temperature gradient inside the chain, as shown in Fig. 1 (circles), and we have verified that the system obeys the Fourier law of heat conduction. For fixed temperatures $T_{1}$ and $T_{2}$, and a chain length varying from $N=64$ to $N=1024$ the heat flux evolves as $1 / N$ as expected for a system with a well defined, finite, thermal conductivity.

We now divide the chain between the thermostats in three regions in which $D_{n}$ takes different values. In the left and right regions, $n=1, \ldots(N-M) / 2$ and $n=(N+M) / 2+1, \ldots, N$, respectively, we set $D_{n}=D$, while in the $\mathrm{M}$ sites of the central region $D_{n}=D_{1}$; 
$\alpha_{n}=\alpha$ for the whole chain. Typically, in our simulations, we take $D=0.5, \alpha=1, K=0.3, m=1, N=128$, $L=16$ and $M=8$ while $D_{1}$ has been varied from 0.5 to 1.2 . As shown by the numerically computed temperature profiles of Fig. 1, the results are now strikingly different. The temperature profile changes as $D_{1}-D$ is increased until, for large enough $D_{1}$, the central region behaves as an insulator, the left and right regions being thermalized at $T_{1}$ and $T_{2}$ respectively, and the heat flux drops to negligible values $\sim 10^{-5}$. Therefore the change in $D_{1}$ can induce a conductor-insulator transition confirmed by Fig. 2 which shows that the averaged heat flux $J$ decreases by two orders of magnitude when $D_{1}-D$ increases from 0 to 0.7 .

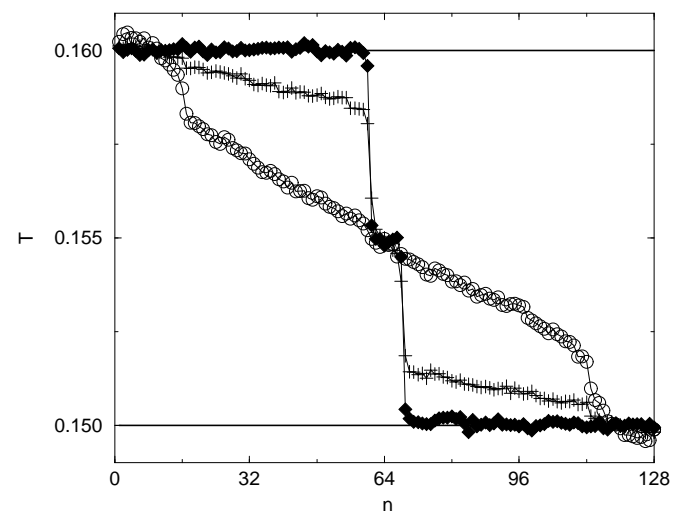

FIG. 1. Temperature profile of the chain with parameters $D=0.5, N=128, \alpha=1, K=0.3, M=8$, and $T_{1}=0.16$, $T_{2}=0.15$. The Morse potential constants are $D_{1}=D=0.5$ - homogeneous system- (circles), $D_{1}=1.2$ (diamonds) and $D_{1}=0.8(+)$

In order to understand this phenomenon, let us consider a linearized version of model (1), which is obtained by linearizing the Morse potential around $y_{n}=0$. The Hamiltonian now writes:

$$
H=\sum_{i=n, N} \frac{p_{n}^{2}}{2 m}+\tilde{D}_{n} y_{n}^{2}+\frac{1}{2} K\left(y_{n}-y_{n-1}\right)^{2}
$$

where $\tilde{D}_{n}=D_{n} \alpha_{n}^{2}$ and $D_{n}, \alpha_{n}$ are defined above.

Numerical simulations with Hamiltonian (2), show that this system displays the same conductor-insulator behavior in a clearer and sharper way. Indeed, due to integrability, there are some typical pathologies, such as the presence of plateaus instead of gradients in the temperature profile, as predicted by [19]. This is why the transition from conducting to insulating regime is even sharper than in the nonlinear case (Fig. 2 (circles) ) until a cut-off is reached.

The equations of motion for the system (2), without thermostats, have plane wave solutions, $y_{n}(t)=e^{i k n-i \omega t}$, where the frequency $\omega$ and momentum $k$ satisfy the dispersion relation $\omega^{2}=2 K+2 \tilde{D}-2 K \cos k$ so that the allowed frequencies are included in the so called phonon band $2 \tilde{D} \leq \omega^{2} \leq 2 \tilde{D}+4 K$.

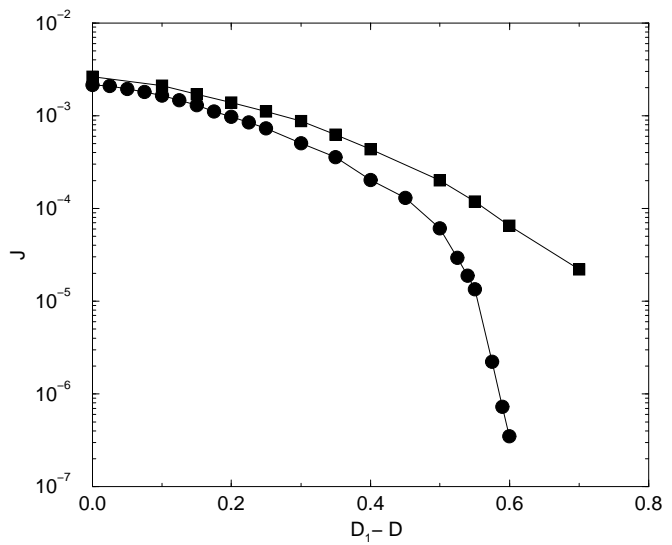

FIG. 2. Heat flux as a function of parameter $D_{1}-D$; the squares refer to the Morse potential, while the circles refer to the corresponding harmonic limit. Here $N=128, D=0.5$, $K=0.3, \alpha=1, L=16$

Let us now consider the harmonic and inhomogeneous chain, with $\tilde{D}_{1} \neq \tilde{D}$ in the central region. In order to propagate through the system a wave should satisfy both dispersion relations $\omega^{2}=2 K+2 \tilde{D}-2 K \cos k$ and $\omega^{2}=2 K+2 \tilde{D}_{1}-2 K \cos k_{1}$. If $\tilde{D}_{1} \neq \tilde{D}$ only a fraction of the allowed frequencies of the waves coming from the left or right regions can satisfy both dispersion equations with real $k_{1}$, and therefore also propagate through the central region. For the remaining frequencies, the second equation can only be solved by imaginary $k_{1}=i \tilde{k_{1}}$ which gives rise to states $y_{n} \sim e^{-\tilde{k_{1} n}}$ which decrease exponentially from the edges of the central region and therefore cannot propagate through it so that their contribution to the total heat flux $J$ is exponentially small. By increasing the separation of the two phonon bands $\tilde{D}_{1}-\tilde{D}$, the fraction of frequencies which can give rise to plane waves inside the entire system falls down and reaches zero as $\tilde{D}_{1}-\tilde{D}>2 K$, where the band separation is larger than the band width, and the bands do not overlap anymore. In this situation, waves coming from the thermostats are reflected back from the central region, the heat flux is exponentially small and the system acts as an almost perfect insulator. In the case of partially overlapping bands one obviously expects an intermediate situation. The dependence of the heat flux on the band overlapping is shown by the lower curve in Fig. 2 and its variation can be calculated with the above analysis.

The addition of a nonlinear term to the Hamiltonian does not change the substance of the above argument. The numerically observed smoother decay of the heat flux vs. $D_{1}-D$ given by the upper curve in Fig. 2 is due to the fact that plane waves are not exact solutions for the nonlinear case and the presence of solitary excitations like breathers may play an important role.

It is now interesting to investigate whether one can control the transport properties of the chain by acting on the temperature of the heat baths without modifying the 
parameters of the interaction or of the on site potential. To this end we recall that, at constant $T$, the nonlinear model can be approximated by a fully harmonic Hamiltonian, with temperature dependent parameters $\Omega^{2}(T)$, $\Phi(T) 13$

$$
H_{0}=\sum_{n=1, N} \frac{p_{n}^{2}}{2 m}+\Omega^{2}(T) y_{n}^{2}+\frac{1}{2} \Phi(T)\left(y_{n}-y_{n-1}\right)^{2}
$$

An effective-phonon analysis, shows that, while $\Phi(T)=K$ is temperature independent, $\Omega(T)$ decreases as $T$ increases 13 . The lowering of the band vs. the temperature is of course determined by the parameters of the system, $\alpha_{n}, D_{n}$. Typical band profiles are shown in the inset of Fig. 3, for different parameter choices.

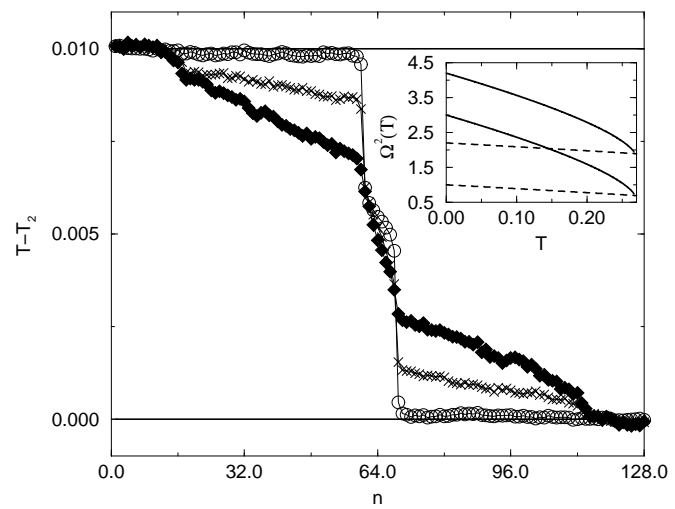

FIG. 3. Temperature profiles for system (11) at three different values of $T_{2}$ for the same temperature gradient $T_{2}-T_{1}$. Parameters are $N=128, M=8, \alpha_{1}=2, \alpha=1, D_{1}=0.375$, $D=0.5 ; T_{2}=0.09$ ( circles), $T_{2}=0.19(\mathrm{x})$ and $T_{2}=0.29$ (diamonds).

Inset: Phonon band profiles vs. temperature; the continuous lines represent the phonon band for $\alpha=2, D=0.375$; the dashed lines $D=0.5, \alpha=1$. The full phonon band is shown with bandwidth $4 K=1.2$

We may now choose parameters $\alpha=1, D=0.5$ (dashed lines in the inset of Fig. (3)) for the left and right regions, and $\alpha_{1}=2, D_{1}=0.375$ (full line in the inset of Fig. (3)) for the central region. From the inset of Fig. (3) it is clearly seen that, at low $T$, the phonon band of the central region does not overlap with the band of the other two side regions. In this situation, the heat flux is nearly zero and the temperature profile shows a clear insulating behavior, with first region thermalized at temperature $T_{1}$ and third region at temperature $T_{2}$ as shown in Fig. 3 (circles). As we increase both temperatures $T_{1}$ and $T_{2}$, keeping $\left|T_{1}-T_{2}\right|$ constant, the bands start to overlap, the temperature profile exhibits a gradient and the heat flux increases drastically (Fig. 3).

As temperature increases, nonlinearity becomes more and more important and the effective phonon analysis becomes less and less meaningful. Nevertheless, numerical analysis shows that the above argument of band overlap still remains valid even if a clean thermal gradient is not obtained and therefore the system does not become a perfect conductor.

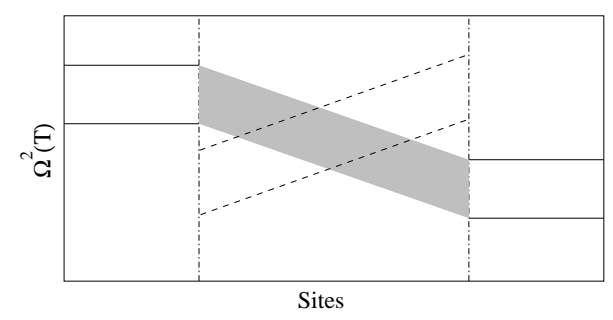

FIG. 4. Schematic picture of the phonon bands in a "thermal rectifier" for two directions of the temperature gradient. The bands in the left and right weakly anharmonic regions do not change significantly with the orientation of the gradient. In the central part, when the high temperature side is on the right, the band evolves in space as shown by the shaded region, while, if the high temperature side is on the left, the band evolves according to the dashed lines.

Once the general mechanism of the thermal conduction in a composite nonlinear lattice has been understood, this opens many possibilities. For instance one can design a thermal rectifier, as schematized on Fig. A. A strongly nonlinear region is sandwiched between two weakly anharmonic left and right domains. In the presence of a thermal gradient in the central part, the effective phonon frequencies evolve in space in a way that depends on the orientation of the gradient. This can provide either a good matching of the bands at the interfaces, leading to a thermal conduction across the system, or a complete mismatch leading to poor conduction. This picture is oversimplified for various reasons: the self-consistent phonon method is approximate, there is not a homogeneous gradient in the system due imperfect thermal contact at the interfaces, and, in spite of nonlinear mixing, in the conducting direction, the frequencies injected at one side of the central region do not shift gradually, following the phonon band, to match the band of the other outer region.

In spite of these restrictions Fig. 5 shows that it is possible to build a simple thermal rectifier where the thermal flux changes by a factor of about 2 when the direction of the thermal gradient is reversed. In fact thermal rectifiers are already known: a layer of fluid, heated from below or from above yields very different thermal flows because the former case leads to convection and the latter does not. To our knowledge, it is nevertheless the first time that a "solid system" (a highly simplified model of a solid indeed) shows such a behavior.

In this work we have studied a specific nonlinear lattice but the results rely on ideas of general validity, the matching of phonon bands or the frequency shift associated to nonlinearity, so that one can expect that various physical systems could exhibit similar behaviors. The simple model that we have chosen to introduce these 
ideas involves an on-site potential which describes the effect of an external substrate not explicitely included in our calculations. In an actual device, such a substrate would introduce a second channel for thermal energy transport, operating in parallel with the channel studied here. This could reduce the efficiency of the rectifier, unless the thermal conductivity of the substrate is significantly lower than the conductivity of the lattice of nonlinear oscillators. Any solid thermal insulator or weak conductor, can, in principle, be used as a substrate. A prototype could be a disordered harmonic lattice [11].

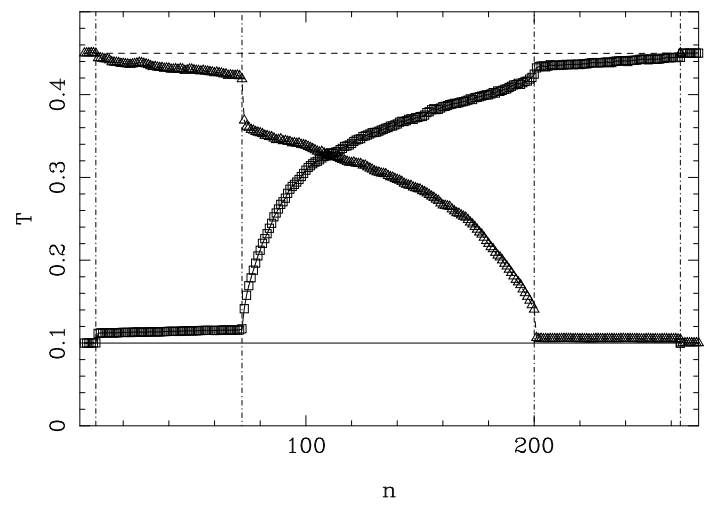

FIG. 5. Temperature profiles in a "thermal rectifier" for two opposite orientations of the thermal gradient. The dash-dotted lines show the borders of the different regions in the lattice. The thermostats have a size $L=8$, the central region a size $M=128$, and the left and right regions contain 64 particles. The coupling constant is $K=0.18$, and the parameters of the Morse potential are $D=4.5, \alpha=0.5$, $D_{1}=0.7, \alpha_{1}=1.4, D^{\prime}=2.8, \alpha^{\prime}=0.5$ in the left, central and right regions respectively. The temperatures of the thermostats are $T=0.1$ and 0.45 . When the high temperature is on the right side of the lattice, the average flux is $J=0.14610^{-3}$ while, when the thermal gradient is reversed the flux drops to $J=0.75510^{-4}$. Note in this case the discontinuities at the interfaces between region which attest of the bad energy transfer at these points.

Among the possible applications of this study, one can think of biological molecules. Controlling the energy flow in biomolecules is important, for instance when the energy of ATP hydrolysis is released locally in a molecular motor to be used elsewhere after some delay [20]. Electronic states could be involved, but we show here that a control of the flow of thermal energy can also be achieved through the strong nonlinearities which exist in biological molecules and could even be tuned by events such as a conformational change. Experiments show that the energy flow in proteins can be extremely slow [21], providing a weakly conducting substrate. On the other hand our results show that a controlable conducting channel attached to this substrate is conceivable. Whether biomolecules exploit this possibility is still a completely open problem.

M.T. thanks the Laboratoire de Physique of ENS Lyon, where part of this work has been performed, for its hospitality and computing facilities. M.P. would like to thank B. Castaing (ENS Lyon) for helpful discussions. This work has been partially supported by EU Contract No. HPRN-CT-1999-00163 (LOCNET network) and by MURST (Prin 2000, Caos e localizzazione in Meccanica Classica e Quantistica).

[1] R.E. Peierls, Quantum theory of solids, Oxford University Press, London (1955).

[2] F.Bonetto, J.L.Lebowitz and L. Ray Bellet in A. Fokas, A. Grigoryan, T. Kibble and B. Zegarlins Eds., Mathematical Physics 2000, Imperial College, London 2000, and math-phys/0002052

[3] G.Casati, J.Ford, F.Vivaldi and W.Visscher, Phys. Rev. Lett. 52, 1861 (1984)

[4] T.Prosen and M.Robnick, J.Phys.A: Math. Gen.,25, 3449 (1992)

[5] S.Lepri, R.Livi and A. Politi, Europhys. Lett, 43, 271, (1998), Physica D119, 3541, (1999), Phys. Rev. Lett., 78, 1896 (1997)

[6] T.Hatano, Phys.Rev.E 59, R1 (1999)

[7] T.Prosen and D.K. Campbell, Phys. Rev. Lett. 84, 2857 (2000)

[8] C. Giardiná, R. Livi, A. Politi and M. Vassali, Phys. Rev. Lett. 84, 2144 (2000)

[9] O.V. Gendelman and A.V. Savin, Phys. Rev. Lett. 84, $2381(2000)$

[10] B.Hu, B.W. Li and H.Zhao, Phys. Rev. E 61, 3828 (2000)

[11] S. Lepri, R. Livi and A. Politi, cond-mat/0112193

[12] M.Peyrard and A.R. Bishop, Phys. Rev. Lett. 62, 2755 (1989)

[13] T. Dauxois, M.Peyrard and A.R. Bishop, Phys. Rev.E 47, 684 (1993)

[14] N. Theodorakopoulos, T.Dauxois and M.Peyrard, Phys.Rev.Lett. 85, 6 (2000)

[15] S. Nosé, J.Chem. Phys. 81, 511 (1984), W.G.Hoover, Phys.Rev.A 31, 1695 (1985)

[16] G.Martyna, M.L.Klein and M.Tuckerman, J. Chem. Phys. 97, 2635 (1992)

[17] W.H. Press, S.A. Teukolsky, W.T. Vetterling and B.P. Flannery, Numerical Recipes, Cambridge University Press (1992)

[18] B.Hu, B.Li and H.Zhao, Phys.Rev.E, 57, 2992 (1998)

[19] Z.Rieder, J.L.Lebowitz and E.Lieb, J. Math. Phys. 8, 1073 (1967); A.Casher and J.L.Lebowitz, J. Math. Phys. 12, 1701 (1971); G.Casati, Il Nuovo Cimento 52B, 257 (1979)

[20] K. Kitamura, M. Tokunaga, A.H. Iwane and T. Yanagida, Nature 397129 (1999)

[21] P. Li and P.M. Champion, Biophysical Journal 66 430436 (1994) 